\title{
Arising From a Published Source Beam, Magnetic and Gravitational Fields Other Than Particle Passes Etc... Then The Beam Is Converted Into a Magnetic Beam
}

\section{Vikrant}

\section{Introdaction}

Light interference, diffraction and polarisatrion events etc, based on the successful delivery of the light wave can be represented nature. Other events, such as some of the light - the Photoelecticlight effect, Compton effect and the Raman effect etc, to reflect such events require Einstein quantum theory. Which acts as particles of light called a photon. Accordingly, acts like a particle beam to see if the publish. If a published ray source (E.g. - bulbs, sun, etc.) occurs, then the beam in the presence of atmospheric molecules, microscopic molecule, particle and planet etc, comes into contact changes its nature and properties. And other properties of the beam starts to appear, it seems to behave like a magnetic beam.

\section{Published beam changes in magnetic beam}

When a source (E.g. - Sun, bulbs, etc.) arising from a published beam starts to move in after all universally. Arising from sources we see a ray of light on (it regardless of any beam get moving in that direction). It is present in the atmosphere, such as beam - molecule, microscopic particle and the planet etc, magnetic and gravitational fields that flow. (As we know. That every small and large particle and the planet etc, helps to separate the gravitational and magnetic field). Then change its properties and nature sounds.

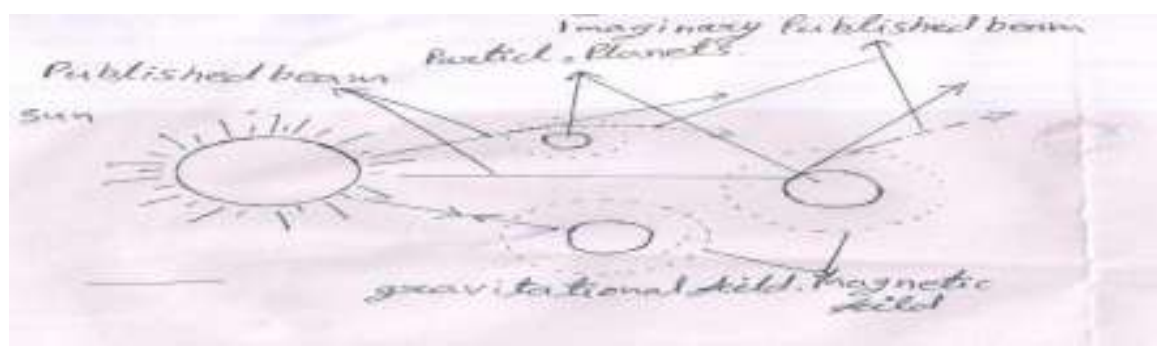

[Fig.(1)]

According to quantum theory Einstein on, light behaves like a particle, the beam of light acts like a particle. Electron which are present. Published beam when the molecules and planets, etc., and from the middle of the gravitational and magnetic fields emanating from it. The complex interaction process between electron in it goes. electron present in the beam that is a fall in the direction of rotation. And seem to spin in the same direction. Which lie within a microscopic-sized atomic become electron is present. Called the domain. This process is very slow moving. Begins with the last part of the beam, Like - like another planet and particle beam, etc. entering the magnetic and gravitational field inside looks, and be in it grows the size of the domain.And gradually a magnetic beam to beam looks. This procedure is possible. When a published beam molecules and planets, etc., etc., passes through the magnetic and gravitational fields. Like - like beam inside the gravitational or magnetic fields, etc. (i.e. - molecules to enter and look toward the planet) the magnetic field inside the beam increases. As the particle beam and the planet, etc., are out of the gravitational and magnetic fields. Then the process stops at the same moment, as no other molecules and planets, etc., come in his way, then it starts apply again. Then the process stops at the same moment, as no other molecules and planets, etc., that come his way, the process (where the same stops) apply again starts.

Example: When a bulb burns a mountain. Published rays radiated from him, (for one beam), the Earth's gravity and microscopic particles present in the atmosphere passes through the gravitational field. (Note: - Each particle has its own gravitation field) Published in the beam is converted into a magnetic beam. This is only possible. While the magnetic dipole moment of the electron present nearby, in the absence of external magnetic field is aligned in the same direction. Published resulting beam size within a microscopic fields become. Beam as published - is as far from its source, by the way - the way that the magnetic dipole moment of the present electron gets the same direction. Publish the resulting beam is converted to a magnetic beam. He published the source from which the beam passes. After fixing a certain distance from its source, (i.e. when the beam is 
converted into a magnetic beam) From that point it stops showing the source. Published Continued rays emanating from the source is.

\section{1:- Magnetic force acting on the beam}

When published beam, particle and other planets, etc., passes through the magnetic and gravitational fields. Then the particle beam by a force acts. The particle and the planet, etc., is known as the gravitational force. The forces present in the beam direction of rotation electron has come. Seem to spin and electron. Which is published in the beam generated electromagnetic torque. Published beam and small - sized domains seem to be infinitesimally small. These forces also affects the speed of the beam.

\section{2:-Electrons published in the spin beam}

According to quantum Einstein published acts like a particle beam. Which is made up of atoms, which is present electron. so that a current loop or an orbital revolution behave like magnetic dipoles. It generates the magnetic moment. Electron is rotating on its axis in addition to its orbital motion. Called the spin.Therefore published magnetic moment in the beam, is generated from the orbital revolution and spin. But most of it is caused by the spin of Electron.

\section{3:- Beam to be published in the domain of}

If you behave like a particle beam, which is made up of. Electron which is present. Which a planet etc, magnetic and gravitational fields that flow. This result seems to be some interaction. And electron seem to spin in the same direction. Permanent magnetic moments inside the beam are generated. Published beam starts to behave like a bar magnet. This minimal size of the list, which makes the effective magnetic field. Is called the domain. Published beam - such as the magnetic field increases from low magnetic field. Increasing the size of the domain. Therefore, the magnetic field increases as the beam. Going to be published in the beam domain, in the absence of external magnetic and gravitational field is scattered by the irregular ways, their resultant magnetic moment in any direction is zero.
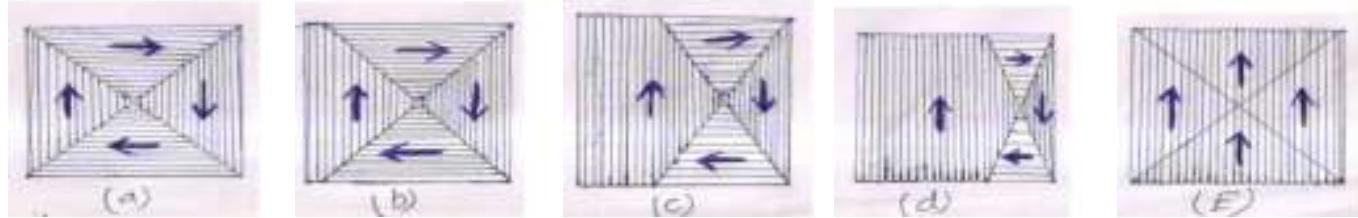

[Fig.(2)]

\section{Speed and path of the beam}

After a published beam emanating from the source, that a particle and a planet, etc., enters the magnetic and gravitational fields. Whose magnetic field is produced in the beam. These magnetic field, which is caused by, acts in the same direction. So the particle and the planet, etc., seems to move faster. That is particle by a force acts on it, and which attracts your own beam. Therefore the speed of light, with the move - with rises. If this planet and the particle beam, etc., is lost from the top (i.e. - magnetic and gravitational field from mid) then the particle beam and the planet, etc. the magnetic and gravitational field looks out toward the exit, the beam speed decreases. Therefore planet and particle etc, is opposed to the motion. decreases the speed of the beam. However, due to published speeds of the beam, the beam speed is very light - which makes a difference. Which is not visible.

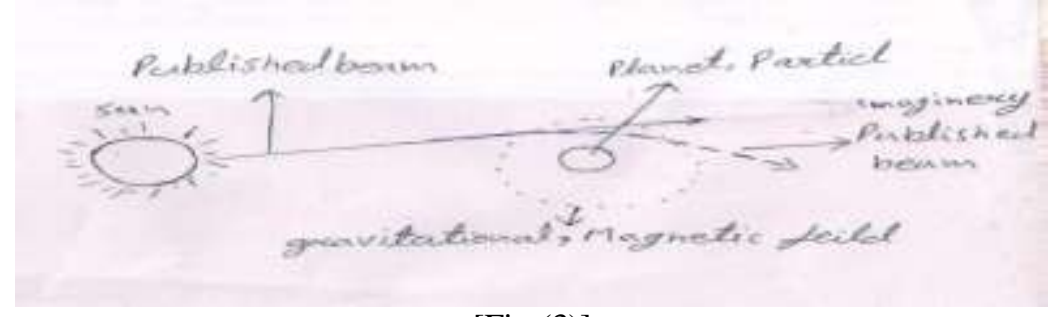

[Fig.(3)]

Published after the beam emanating from the source starts to speed, the beam particle and a planet, etc., between the magnetic and gravitational field passes. (Planet and particle etc., above or below) Which is published in the beam generated magnetic field, the magnetic field acts in the same direction. Which is caused 
by. That is particle by a force acts on it, and which attracts your own beam. Therefore the speed of light, with the move - with rises. Therefore, it seems to attract published beam. Beam path which is like a parabola. [As shown in fig. (3)]

Example:-Hidden sun, and the sun comes out etc, [Prrthvi before sunrise because the light is on. And after hiding the sun light for a long time tend to have Prrthvi.]

\section{IV. mathematical proved}

When a published ray source is generated. Then starts to speed. Generated a beam of rays, as mass M, and the $\mathrm{V}$ velocity is moving in the environment. Believed to arise from the beam source, then $\mathrm{E}_{\mathrm{p}}$ is the electric field. (Where $\mathrm{E}_{\mathrm{p}}$ - electric field of Published beam) This is considered a particle beam and the planet, etc., passes through the magnetic and gravitational fields. Resulting beam is generated in the magnetic field, the magnetic field $\mathrm{B}_{\mathrm{p}}$ is considered to occur within the beam.(Where $\mathrm{B}_{\mathrm{p}}$-magnetic field of published beam). When a particle beam, etc., passes through the magnetic field, then it seems to work a force $\mathrm{F}_{\mathrm{g}}\left(\right.$ Where $\mathrm{F}_{\mathrm{g}}$-The gravitational force acting on the particle beam). Beam which attracts your behalf.Then the particle beam, etc., due to the magnetic field of the magnetic field in the beam,

$$
\mathrm{B}_{\mathrm{p}}=\mathrm{MVL} \quad\left(\mathrm{F}_{\mathrm{g}}+\mathrm{L}\right)
$$

electric field $\mathrm{E}$ is published beam. Considered in the present $\mathrm{e}$, which $\mathrm{v}$ is the velocity of the beam moving inside. Then beam the electric field,

$$
\mathrm{E}_{\mathrm{p}}=\mathrm{ev} / \mathrm{L}^{2}
$$

Where $\mathrm{L}$-is the distance between the source and the beam

$$
\mathrm{E}_{\mathrm{p}} \mathrm{L}^{2}=\mathrm{ev}
$$

This is considered a particle beam, etc., passes through the magnetic and gravitational fields, which at that, part of the gravitational force $F_{g}$ to act and think. Beam, which is opposed to the electric field. But acts in the direction of the beam in the present electron etc.Then from equation (1),

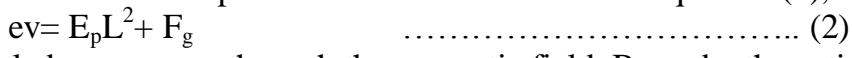

If a particle beam passes through the magnetic field. Beam by the action against the particle,

$$
\mathrm{W}=\mathrm{F}_{\mathrm{g}} \mathrm{L}
$$

Beam when published, a particle etc, magnetic and gravitational field moves. Then a force acts on the beam path of the light - looks like a parabola. Then centripetal force acting on,

$$
\mathrm{F}=\mathrm{MVW}
$$

$$
\begin{aligned}
& \text { Note }:-M V W=F=M V^{2} / r=M V V / r=M V a t / r \quad \text { if } a=V / t \quad V=r / t \\
& =M V a / V=M a=M V W
\end{aligned}
$$

From equation (3), we get

$$
\mathrm{F}=\mathrm{MV} \mathrm{F}_{\mathrm{g}} \mathrm{L}
$$

By another particle, magnetic and gravitational field acting on the beam,

$$
\mathrm{B}_{1}=\mathrm{F} / \mathrm{ev}
$$

From equation (2) and (4), we get

$$
\mathrm{B}_{1}=\mathrm{MVLF}_{\mathrm{g}} /\left(\mathrm{E}_{\mathrm{p}} \mathrm{L}^{2}+\mathrm{F}_{\mathrm{g}}\right)
$$

$\mathrm{L}$ distance of the beam from the source, as the beam is moving from the atmosphere $\mathrm{V}$ Beg. Acceleration acting on the beam,

$$
a_{\mathrm{g}}=\mathrm{VL}
$$

If a particle beam, etc., is moving in the magnetic and gravitational fields. Then the force acting on the beam,

$$
\begin{aligned}
& F_{g=M} a_{g} \\
& F_{g=M V L}
\end{aligned}
$$

A published beam, a particle passes through the magnetic field, etc. That a force is. This serves to generate the magnetic field in the beam. Therefore act to generate the magnetic field in the beam,

$$
\mathrm{W}=\mathrm{F}_{\mathrm{g}} \mathrm{L}
$$

From equation (7), and (8), we get

$$
\mathrm{W}=\mathrm{MVL}^{2}
$$

When the magnetic field is produced inside the beam. Then a force acts on the beam electrons. Electron which are in the direction of rotation. Hence the task to generate the magnetic field in the beam and the force acting on the beam electrons is equal to each other.

$$
\begin{gathered}
\mathrm{W}=\mathrm{F} \\
\mathrm{F}=\mathrm{MVL}^{2}
\end{gathered}
$$


Hence, the magnetic field $\mathrm{B}_{2}$ generated inside the beam, then

$$
\mathrm{B}_{2}=\mathrm{F} / \mathrm{ev}
$$

From using equation (2) and (10)

$$
\mathrm{B}_{2}=\mathrm{MVL}^{2} /\left(\mathrm{E}_{\mathrm{p}} \mathrm{L}^{2}+\mathrm{F}_{\mathrm{g}}\right)
$$

Published a beam, a particle, etc., passes through the magnetic and gravitational fields. Magnetic moment in the beam is generated. Published resulting total magnetic field within the beam, the beam projected onto the vector sum of the magnetic field and the magnetic field is generated. From equation (6) and (12),Then

$$
\begin{gathered}
\mathrm{B}_{\mathrm{p}}=\mathrm{B}_{1}+\mathrm{B}_{2} \\
\mathrm{~B}_{\mathrm{p}}=\mathrm{MVLF}_{\mathrm{g}} /\left(\mathrm{E}_{\mathrm{p}} \mathrm{L}^{2}+\mathrm{F}_{\mathrm{g}}\right)+\mathrm{MVL}^{2} /\left(\mathrm{E}_{\mathrm{p}} \mathrm{L}^{2}+\mathrm{F}_{\mathrm{g}}\right)
\end{gathered}
$$

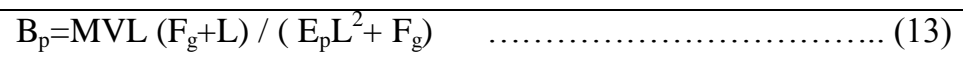

Proved that. Using this formula, magnetic and gravitational field of a particle, etc. Due to a study published in the beam to the magnetic field is generated.

As arising from a published source beam, after being hit by an object or will change, he moves to another Direction or the Direction. Therefore beam collided, destroying some of his energy. Or architectural absorbed by is taken. Believed to absorb the energy $\mathrm{E}$, then

$$
\mathrm{B}_{\mathrm{p}}=-\left[\mathrm{MVL}\left(\mathrm{F}_{\mathrm{g}}+\mathrm{L}\right) /\left(\mathrm{E}_{\mathrm{p}} \mathrm{L}^{2}+\mathrm{F}_{\mathrm{g}}\right)-\mathrm{E}\right]
$$

Where, $B_{\mathrm{p}}{ }^{-}$-is the magnetic field of reflection published beam

Change the object hit by the ray changes in the same direction, the direction it comes from. Beam collide and change the source is coming from. Then energy from the receiving end. Adding equations (13) and (14), we get

$$
\begin{aligned}
& \mathrm{B}=\mathrm{B}_{\mathrm{p}}+\mathrm{B}_{\mathrm{p}}{ }^{\prime} \\
& \left.\mathrm{B}=\mathrm{MVL}\left(\mathrm{F}_{\mathrm{g}}+\mathrm{L}\right) /\left(\mathrm{E}_{\mathrm{p}} \mathrm{L}^{2}+\mathrm{F}_{\mathrm{g}}\right)+\left\{-\left[\mathrm{MVL}\left(\mathrm{F}_{\mathrm{g}}+\mathrm{L}\right) / \mathrm{E}_{\mathrm{p}} \mathrm{L}^{2}+\mathrm{F}_{\mathrm{g}}\right)-\mathrm{E}\right]\right\} \\
& \left.\mathrm{B}=\mathrm{MVL}\left(\mathrm{F}_{\mathrm{g}}+\mathrm{L}\right) /\left(\mathrm{E}_{\mathrm{p}} \mathrm{L}^{2}+\mathrm{F}_{\mathrm{g}}\right)-\mathrm{MVL}\left(\mathrm{F}_{\mathrm{g}}+\mathrm{L}\right) /\left(\mathrm{E}_{\mathrm{p}} \mathrm{L}^{2}+\mathrm{F}_{\mathrm{g}}\right)+\mathrm{E}\right] \\
& \mathrm{B}=\mathrm{E}
\end{aligned}
$$

Hence it is clear; the energy of the rays generated by the two rays is equal to the magnetic field.

Example: - When a person or object, etc., we are in the light of the sun. Then a ray of light shines on it. Rays, some of which are hit by an object or person is changed. Content and altered by humans and sun rays collide with the incoming beam is published. And those originating from the energy, the energy is felt around the object or person.

As such: - the winter sun can also humans,

Special condition:-

(a):- Published on the beam, not from any other particle and the magnetic field passes. Therefore the gravitational force, $\mathrm{F}_{\mathrm{g}}$ acting on the beam does not. Or $\mathrm{F}_{\mathrm{g}}=0$ from equation (12),

$$
\begin{aligned}
& \mathrm{B}_{\mathrm{p}}=\mathrm{MVL}(0+\mathrm{L}) /\left(\mathrm{E}_{\mathrm{p}} \mathrm{L}^{2}+0\right) \\
& \mathrm{B}_{\mathrm{p}}=\mathrm{MVL} / \mathrm{E}_{\mathrm{p}} \mathrm{L}^{2} \\
& \mathrm{~B}_{\mathrm{p}}=\mathrm{MV} / \mathrm{E}_{\mathrm{p}}
\end{aligned}
$$

Hence, there will be no change in beam published. Whether beam from the source drive goes finalized. Therefore would not generate the magnetic field in the beam.

(b):- Infinite distance from its source to decide the published beam, and on the planet as any other particle, etc., and the gravitational field caused by the magnetic force on the functions are continuous, the magnetic field of the beam is infinite .If $\mathrm{L}=$ infinite;

From equation (12)

$$
\begin{gathered}
\mathrm{B}_{\mathrm{p}}=\mathrm{MVL}\left(\mathrm{F}_{\mathrm{g}}+\mathrm{L}\right) /\left(\mathrm{E}_{\mathrm{p}} \mathrm{L}^{2}+\mathrm{F}_{\mathrm{g}}\right) \\
\mathrm{B}_{\mathrm{p}}=\text { Infinite }
\end{gathered}
$$

Therefore, the magnetic field becomes infinite infinite ray drive. This is possible only when the magnetic and gravitational fields, etc. that a particle passes through.

\section{V. characteristics of published beam}

5.1:-Advantages: (a):-When the beam, magnetic and gravitational field of another particle enters etc. Then a force acts on the particle and planet. Which light beam path - in the shape of a parabola becomes.

(b):- When the beam, magnetic and gravitational field of a particle and B, enters the beam speed rises. However originates. Then decreases.

(c):- Beam that can be derived from any published source, constant magnetic and gravitational fields of the other particle is passing through. Then the beam is converted into a magnetic beam. 
5.2:-Limitations: (a):- Published on the beam, not from any other particle and the magnetic field passes. Therefore the gravitational force, $\mathrm{F}_{\mathrm{g}}$ acting on the beam does not. Or $\mathrm{F}_{\mathrm{g}}=0$ from equation (12),

$\mathrm{B}_{\mathrm{p}}=\mathrm{MVL}$
$\mathrm{B}_{\mathrm{p}}=\mathrm{MVL}^{2} / \mathrm{E}_{\mathrm{p}} \mathrm{L}^{2}$

$\mathrm{B}_{\mathrm{p}}=\mathrm{MVL}(0+\mathrm{L}) /\left(\mathrm{E}_{\mathrm{p}} \mathrm{L}^{2}+0\right)$

$\mathrm{B}_{\mathrm{p}}=\mathrm{MV} / \mathrm{E}_{\mathrm{p}}$

Hence, there will be no change in beam published. Whether beam from the source drive goes finalized. Therefore would not generate the magnetic field in the beam.

(b):- Infinite distance from its source to decide the published beam, and on the planet as any other particle, etc., and the gravitational field caused by the magnetic force on the functions are continuous, the magnetic field of the beam is infinite .If $\mathrm{L}=$ infinite;

From equation (12)

$\mathrm{B}_{\mathrm{p}}=$ Infinite

$$
\mathrm{B}_{\mathrm{p}}=\mathrm{MVL}\left(\mathrm{F}_{\mathrm{g}}+\mathrm{L}\right) /\left(\mathrm{E}_{\mathrm{p}} \mathrm{L}^{2}+\mathrm{F}_{\mathrm{g}}\right)
$$

Therefore, the magnetic field becomes infinite infinite ray drive. This is possible only when the magnetic and gravitational fields, etc. that a particle passes through.

(C):- If the beam does not go away any of your sources. Therefore $\mathrm{L}=0$, then the particle and, therefore, the force of the gravitational and magnetic fields there will also not pass. $F=0$, from equation (12), Then, publish beam Electric Field

$$
\mathrm{B}_{\mathrm{p}}=\mathrm{MVL}\left(\mathrm{F}_{\mathrm{g}}+\mathrm{L}\right) /\left(\mathrm{E}_{\mathrm{p}} \mathrm{L}^{2}+\mathrm{F}_{\mathrm{g}}\right)
$$

$\mathrm{E}_{\mathrm{p}}=\mathrm{MVL}\left(\mathrm{F}_{\mathrm{g}}+\mathrm{L}\right) /\left(\mathrm{B}_{\mathrm{p}} \mathrm{L}^{2}\right)+\mathrm{F}_{\mathrm{g}} / \mathrm{L}^{2}$

$\mathrm{E}_{\mathrm{p}}=0$

(d):- When the beam is approximately $10^{-40}$ to $10^{-32}$ meters of published domain.

5.3:-Indicate: (a):-This type of event can be studied only. Published when the beam out of its source, to any other particle and magnetic and gravitational Regional defenses and enters.

\section{Experiment}

6.1:-object: Published in the beam, due to the magnetic field of any other particle, etc., to study the magnetic field generated.

6.2:-Apparatus used: A bulb, battery, two long metal rod inside the upper portion of the blank and the partridge and sliced, two T-shaped fortress, a kilo-meter scale which measures

6.3:-Principl and formula: From any source to generate a beam, after it passes through a particle etc. Whose magnetic field is produced in the beam. It acts in the direction of the magnetic field, which is caused by. This impact on the speed of the beam. But in a magnetized beam is changed.

Published beam generated in the magnetic field,

$$
B_{p}=\operatorname{MVL}\left(F_{g}+L\right) /\left(E_{p} L^{2}+F_{g}\right)
$$

6.4:- Experimental setup: RsTU is a wooden watching. Rs corner which has a kilo meter scale, a measurement of the beam is published. And in the middle of $\mathrm{AB}$ and $\mathrm{CD}$ and found two metal rod is laid parallel to each other. rod $\mathrm{AB}$, nail above the $\mathrm{P} 1$, and the rod $\mathrm{CD}$, above the nail $\mathrm{P} 2$, is placed in such a way that the front and behind the nail may be required. And point G, is put to a bulb, and it has been linked to a battery. Which bulb Then a ray of light bulb out of the published rod CD, there are parallel.As shown in fig.(4.a)

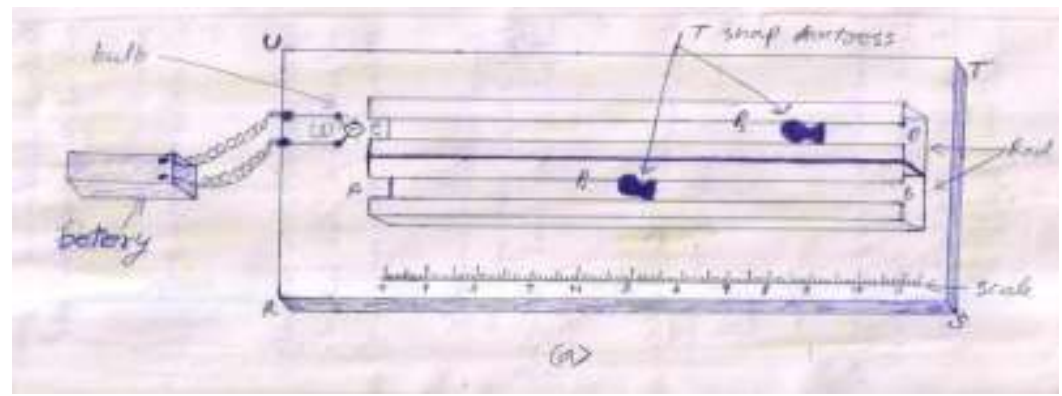

[Fig.(4.a)]

6.5:-working: Current in the battery to the bulb, the bulb published beam is generated. Few rays of which moves parallel to the rod $\mathrm{CD}$. The beam from point $\mathrm{C}$ to point $\mathrm{D}$ moves. Among those present, and minimal particle and magnetic particle passes through the gravitational field. P2 and P2 is supposed to act like a man. $3 \mathrm{~kg}-\mathrm{m}$ away from point $\mathrm{C}$ on the $\mathrm{P} 2$ position,. Published beam emitted from the bulb falls on man standing on $\mathrm{P} 2$. And the source is clearly visible. But - as the distance between P2 and C increases. Anyway - such a source is not clearly visible. Therefore believed to increase the distance between point $\mathrm{C}$ and $\mathrm{P} 2$ give $10 \mathrm{~kg} \mathrm{~m}$. The source 
does not appear when you view the source. Therefore arise from sources not to publish the resulting beam P2, and minimal particle and particle etc, position between point $\mathrm{C}$ and $\mathrm{P} 2$, passes through the gravitational and magnetic fields. Beam resulting in a magnetic field is generated. And such - like the beam gets further away from the source. Namely magnetic and gravitational field is inward. Then move the magnetic field in the beam. Therefore, a magnetic beam is converted to publish beam. Those published in the last part of the light beam in the beam magnetic field starts. And forward it to the magnetic field generated in the beam increases. And there is a situation, the entire beam is generated in the magnetic field. Therefore beam is converted into magnetic beam. And P2 can be seen when viewed from the source.

$5 \mathrm{~kg}-\mathrm{m}$ away from point $\mathrm{C}$ to $\mathrm{P} 2$ to be put on. And P1, $0 \mathrm{~kg}-\mathrm{m}$ drive from the point $\mathrm{C}$ are considered. Published source of the beam moves towards P2. And after hitting the side of P1 is changed. But it is not reaching the beam wins P1. Because of the magnetic and gravitational fields, etc. The particle beam passes through. Beam resulting in a magnetic field is generated. But between point $\mathrm{C}$ and $\mathrm{P} 2$, the process is moving normally. And P2 after hitting some of its energy is lost. (Or P2 is absorbed by.) Which light up the inside of the magnetic field bound to think. The time and beam distance from point $\mathrm{C}$ to the P2 looks after colliding beam that he could not judge the distance. With a few extra moves fast in her field. And beam is converted into a magnetic beam. Therefore, from the point of view point P1 P2 is not there.

If $5 \mathrm{~kg}-\mathrm{m}$ distance from point $\mathrm{C}$ to $\mathrm{P} 2$ have the same status. $3 \mathrm{~kg}-\mathrm{m}$ distance from point $\mathrm{C}$ to $\mathrm{P} 1 \mathrm{but}$ will get it. Then, upon seeing a published source beam moves towards P2. P1 and P2 to move ahead or reflects and looks. When the beam is reached on P1. P1 look, P2 appears. Because the remainder of the electrical part of the beam or the beam is completely polarized beam does not change. Therefore, seen from P1, P2 is clearly visible.

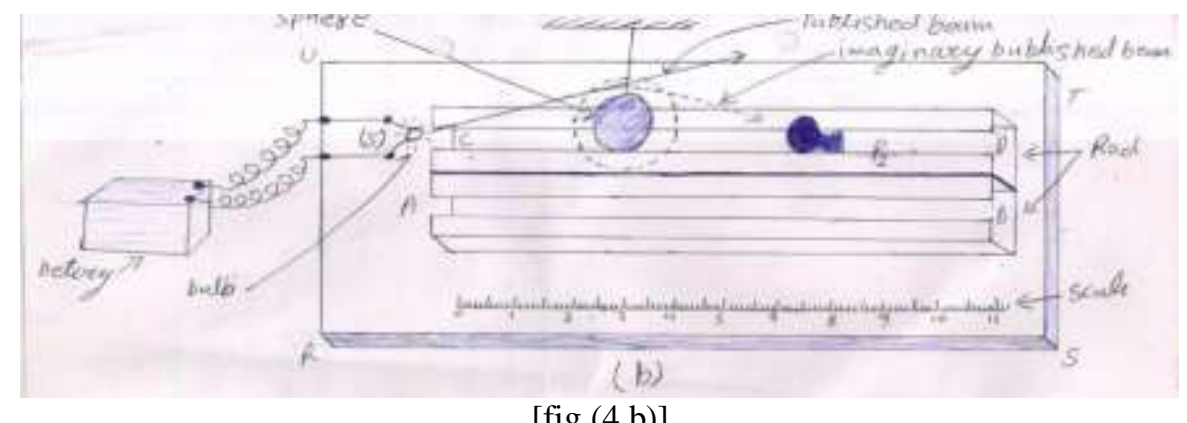

[fig.(4.b)]

The distance from point $\mathrm{C}$ to $\mathrm{P} 2$ to be the case, look at the source is clearly visible. Considered $8 \mathrm{~kg}-\mathrm{m}$ drive position. And put the sphere between point $\mathrm{C}$ and P2. Whose radius $\square$ r' miter. Believed $\square \mathrm{h} \square$ miter surrounded by shells, shells spread is magnetic and gravitational fields. When the battery has to be passed through by a current source. Published by the source beam is generated. Considered any of these shells published beam passes through the magnetic and gravitational fields. Beam resulting in a magnetic field is generated. These acts in the direction of the magnetic field, which is due to be produced. Beam seems to be attracted to and published shells. Seems to work on it a force which attracts your behalf beam . And such - like the beam of the magnetic field inside the sphere rises., And also increases the magnetic field generated inside the beam., And on beam, also increases the force acting through the shells. Because the beam is deflected from its path. And P2 look like a parabolic path of the beam is visible. As shown in [fig.(4.b)],

Published as a ray from the center of the magnetic field not go shells, the shells are straight. Shells in the beam due to the magnetic field, the magnetic field is generated. Towards the region of the same acts for which they are generated. And a force acts on it, which seems to attract the beam on its behalf. Published which rises the speed of light. So when the beam enters the magnetic field of the sphere, then its speed increases., And when the ball is thrown out of the magnetic field of the beam, then its speed decreases., But is light moves so fast, that on that very light - which makes a difference.

6.6:-Result:(a):- Beam that can be derived from any published source, constant magnetic and gravitational fields of the other particle is passing through. Then the beam is converted into a magnetic beam.

(b):- Published the beam, the shells of the magnetic field, as well as the sphere passes through. Beam path that looks more like a parabola. 